\title{
Racial Disparities in Above-knee Amputations After TKA: A National Database Study
}

\author{
Jaiben George MBBS, Suparna M. Navale MS, MPH, Nicholas K. Schiltz PhD, \\ Miguel Siccha MD, Alison K. Klika MS, Carlos A. Higuera MD
}

Published online: 19 December 2016

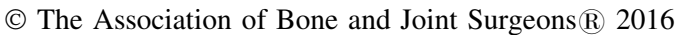

\begin{abstract}
Background Above-knee amputation (AKA) is a rare but devastating complication of TKA. Although racial disparities have been previously reported in the utilization of TKA, it is unclear whether disparities exist in the rates of AKA after TKA.

Questions/purposes (1) Which gender-racial group has the highest rate of AKA from septic and aseptic
\end{abstract}

One of the authors (NKS) has received research support funding from National Institutes of Health Grant KL2TR000440. One of the authors (CAH) has been involved in the following relevant financial activities outside of this work and/or other relationships or activities that readers could perceive to have influenced, or that give the appearance of potentially influencing, this manuscript: KCI (San Antonio, TX, USA), Stryker (Kalamazoo, MI, USA), Myoscience (Fresno, CA,

USA), CD Diagnostics (Claymont, DE, USA), Orthopaedic Research and Education Foundation (Rosemont, IL, USA), Convatec (Greensboro, NC, USA), and Pfizer (New York, NY, USA).

All ICMJE Conflict of Interest Forms for authors and Clinical Orthopaedics and Related Research $\mathbb{R}$ editors and board members are on file with the publication and can be viewed on request.

Each author certifies that his or her institution approved or waived approval for the human protocol for this investigation and that all investigations were conducted in conformity with ethical principles of research.

This work was performed at Cleveland Clinic, Cleveland, OH, USA.

Electronic supplementary material The online version of this article (doi:10.1007/s11999-016-5195-3) contains supplementary material, which is available to authorized users.

J. George, M. Siccha, A. K. Klika ( $₫)$, C. A. Higuera Department of Orthopedic Surgery, Cleveland Clinic, 9500 Euclid Avenue, A40, Cleveland, OH 44195, USA

e-mail: klikaa@ccf.org

S. M. Navale, N. K. Schiltz

Department of Epidemiology and Biostatistics, Case Western Reserve University, Cleveland, OH, USA complications of TKA? (2) Which age groups have higher rates of AKA from septic and aseptic complications of TKA?

Methods Using National Inpatient Sample data from 2000 to 2011, AKAs resulting from complications of TKA were identified using a combination of International Classification of Diseases, 9th Revision procedure and diagnosis codes. Of the 341,954 AKAs identified, 9733 AKAs were the result of complications of TKA (septic complications $=8104$, aseptic complications $=1629)$. Standardized AKA rates were calculated for different age and gender- racial groups by dividing the number of AKAs in each group with the corresponding number of TKAs. Standardized rate ratios were calculated after adjusting for demographics and comorbidities.

Results After adjusting for age and comorbidities, black men had the highest rate of AKA after TKA (adjusted rate in black men $=578$ AKAs per 100,000 TKAs, standardized rate ratio $[\mathrm{SRR}]=4.32$ [confidence interval $\{\mathrm{CI}\}, 3.87$ 4.82], $\mathrm{p}<0.001)$. Black men also had the highest rate of AKA after septic complications of TKA $(p<0.001)$. The adjusted rates of AKA were higher in patients younger than 50 years (adjusted rate $=473, \mathrm{SRR}=3.14$ [CI, 2.94-3.36], $\mathrm{p}<0.001$ ) and older than 80 years (adjusted rate $=297$, $\mathrm{SRR}=1.85$ [CI, 1.76-1.95], $\mathrm{p}<0.001)$.

Conclusions The rising demand for TKA has led to an increase in the number of AKAs performed for complications of TKA in the United States. Although we did not find an increase in the rate of AKA during the study period, certain populations, including black men and patients older than 80 years and younger than 50 years, had higher rates of AKA. Further studies are required to understand the reasons for these disparities and measures should be undertaken to eliminate these disparities.

Level of Evidence Level III, therapeutic study. 


\section{Introduction}

TKA is considered to be one of the most effective procedures in orthopaedic surgery, relieving the suffering of millions of people with arthritis and improving their quality of life $[11,27]$. Despite the documented benefits of TKA, racial and ethnic disparities have been reported in the utilization of TKA in the United States [2, 4]. In addition to the disparities in TKA utilization, studies have also demonstrated disparities with respect to outcomes and complications of TKA [28]. However, these studies were mostly limited to in-hospital complications and patientreported outcomes [16]. Above-knee amputation (AKA) is a rare but serious complication of TKA. The reported prevalence of AKA after TKA ranges from 140 to 410 AKAs per 100,000 TKAs [10, 21]. The functional outcomes after amputation are poor with approximately half of the patients being nonambulatory after TKA-related AKAs $[6,7]$. Although racial disparities in the rates and levels of amputation have been previously described for amputations resulting from vascular reasons, these have been unexplored for amputations arising as a result of complications of TKA $[8,17]$.

Prosthetic joint infection (PJI) poses a challenge to orthopaedic surgeons, because recurrent infections are difficult to treat [18]. Treatment options are limited after multiple failed revision procedures and, unfortunately, AKA sometimes is the best treatment option. Although persistent PJI is responsible for the vast majority of AKAs after TKA, other TKA-related complications like periprosthetic fractures, severe bone/soft tissue loss, and intractable pain can also result in amputations $[10,26]$. Because strategies to prevent AKA will depend on the factors influencing the rates of AKA, it is important to understand the sociodemographic disparities in the rates of AKA after TKA and the contribution of PJI toward AKA. Given the poor functional outcomes and broad societal implications of AKA, information about demographic disparities in AKA is crucial for policymakers and healthcare professionals.

Therefore, we asked: (1) Which gender-racial group has the highest rate of AKA from septic and aseptic complications of TKA? (2) Which age groups have higher rates of AKA from septic and aseptic complications of TKA?

\section{Materials and Methods}

The Nationwide Inpatient Sample (NIS) from 2000 to 2011 was utilized for this study [22]. The institutional review board deemed this study exempt from approval because it used nonidentifiable information obtained from a public source. Diagnosis and procedure information was captured using International Classification of Diseases, 9th Revision, Clinical Modification (ICD-9-CM) codes.

NIS data from 2000 to 2011 were retrospectively queried to identify 341,954 AKAs, defined as discharges with a primary or secondary procedure code of 84.17 (Fig. 1). After initially excluding patients with a diagnosis code for traumatic amputations or lower limb malignancies, patients were grouped into having a diagnosis code for a complication of TKA or a diagnosis code for other causes of AKA (peripheral vascular disease, diabetes, soft tissue infections, etc). Because specific ICD-9 procedure/diagnosis codes for TKA-related AKA were not available, the amputations with a diagnosis indicating a complication of TKA were assumed to be from a failure of TKA (Appendix 1 [Supplemental materials are available with the online version of CORR $\AA$.]).

All AKAs were further classified into "septic" or "aseptic" based on the underlying diagnosis. Septic AKAs include all the procedures with any of the ICD-9 diagnosis codes implying failure from infection of TKA or related components. Aseptic AKAs include the amputations with the ICD-9 diagnosis codes implying failure from causes other than infection such as periprosthetic fractures and dislocations. Patients with multiple diagnosis codes for both septic and aseptic failures were classified as septic. Of the 9733 AKAs, 8104 (83\%) were a result of septic complications. We also validated the results obtained using this approach with the prevalence reported in previously published studies. The prevalence in this study was approximately 170 AKAs per 100,000 TKAs (0.17\%), which was in agreement with other studies reporting prevalence ranging from 140 to 180 AKAs per 100,000 TKAs $(0.14 \%-0.18 \%)$ in the US population [10, 26]. Also, the proportion of AKAs resulting from septic complications in this study (83\%) was similar to that reported in the literature $(76 \%-88 \%)[10,26]$. The detailed description of the ICD-9 codes and the methodology along with the assessments of its validity are provided (Appendix 1).

To obtain standardized rates for age and gender-racial groups, the number of AKAs in each subgroup was divided by the corresponding number of TKAs. Age was divided into four groups: $<50,50$ to 64,65 to 80 , and $>80$ years; and race was divided into three groups: white, black, and others (includes Hispanics, Asians, and missing values). For this study, the Elixhauser score was used to adjust for comorbidities and all patients were divided into three groups based on score: score 0 to 1 , score 2 to 4 , and score $>4$. All standardized rates were multiplied by 100,000 to express the numbers as AKAs per 100,000 TKAs. The septic burden was defined as the percentage of septic AKAs among all AKAs (septic AKA/septic AKA + aseptic AKA). The standardized AKA rate for a particular year was obtained by dividing the number of AKAs in a 
Fig. 1 The flowchart depicts the approach used in this study to identify AKA along with the ICD-9 codes. HCUP = Healthcare Cost and Utilization Project.

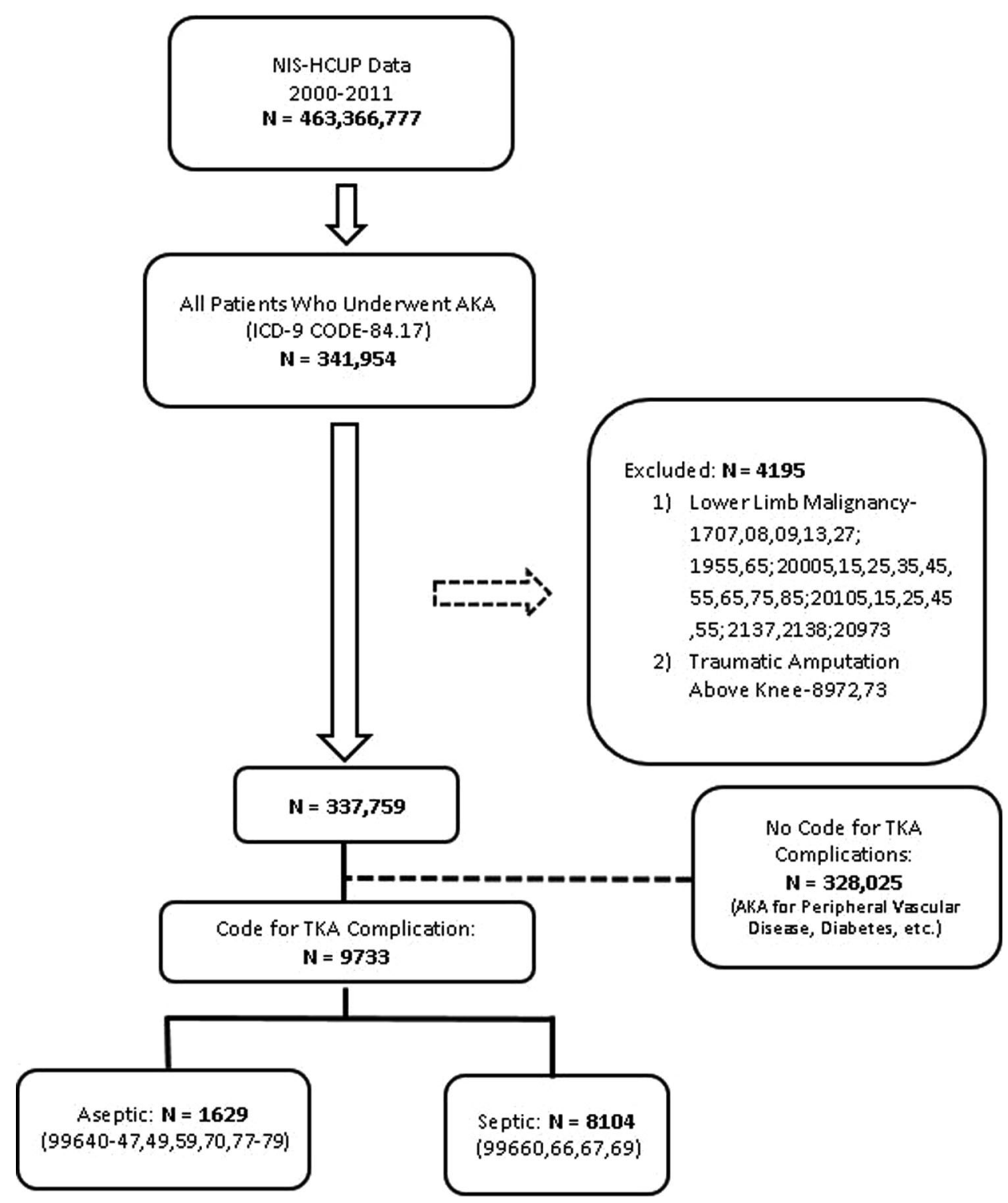

particular year by the number of TKAs in the corresponding year.

\section{Statistical Analysis}

The rates of TKA and AKA were stratified by age, gender, race, and Elixhauser score and adjusted rates were calculated using the largest group as the standard population. For example, to obtain the adjusted AKA rates for the different gender-racial groups, the standardized AKA rate in each gender-racial subgroup, stratified by age and Elixhauser score, was multiplied with the corresponding distribution of TKA among white women. Adjusted rates for various racial groups and age groups were similarly calculated using blacks and age 65 to 80 years as the standard populations. Standardized rate ratios were calculated by dividing the observed number of AKAs in a certain group by the expected number of AKAs based on the standard population. Poisson regression was used to evaluate the yearly changes in the incidence of AKA. Yearly changes in standardized AKA rates and septic burden were evaluated with a simple linear regression model. As a result of the stratified sampling format of the NIS, all frequencies and analyses were converted to national estimates using weights and accounting for the complex sample design.

\section{Results}

Black men had the highest standardized rate of AKA after TKA (black men: 602 AKAs per 100,000 TKAs [469 of 
77,968], black women: 245 AKAs per 100,000 TKAs [586 of 238,939$]$, white men $=222$ AKAs per 100,000 TKAs [3174 of $1,430,020]$, white women $=119$ AKAs per 100,000 TKAs [2782 of 2,347,568], other men $=222$ AKAs per 100,000 TKAs [1365 of 615,698$]$, other women $=121$ [1359 of 1,123,393], $\mathrm{p}<0.001)$. After adjusting for age and comorbidities, black men had the highest rate of AKA (adjusted rate in black men $=763$, standardized rate ratio $[\mathrm{SRR}]=4.71$ [confidence interval $\{C I\}, 4.28-5.18], p<0.001)$. The adjusted rate of AKA after septic complications of TKA was highest in black men (adjusted rate in black men $=578$ AKAs per 100,000 TKAs, SRR $=4.32$ [CI, 3.87-4.82], $\mathrm{p}<0.001)$. The adjusted rate of aseptic AKA was also highest among black men (adjusted rate in black men $=192$ AKAs per 100,000 TKAs, SRR $=6.64$ [CI, 5.47-8.04], $\mathrm{p}<0.001$ ).

Throughout the study period, the rates of AKA remained high in black men (annual range $=382-921$ AKAs per 100,000 TKAs) (Fig. 2). There was no significant decrease in the rates of AKA in any of the racial-gender groups (white men, $\mathrm{p}=0.339$; white women, $\mathrm{p}=0.826$; black men, $p=0.131$; black women, $p=0.084)$. Overall, black patients had the highest rate of AKA after TKA (white = 174 AKAs per 100,000 TKAs [4555 of 2,852,603], black $=$ 422 AKAs per 100,000 TKAs [ 940 of 222,727], others = 178 AKAs per 100,000 TKAs [2337 of 1,312,798], p < 0.001). After adjusting for age, gender, and comorbidities, blacks had the highest standardized rate of AKA (adjusted rate in blacks $=530$, SRR $=2.44[\mathrm{CI}, 2.29-2.60], \mathrm{p}<$ 0.001 ). The adjusted rate of AKA after septic complications of TKA was also highest in blacks (adjusted rate in blacks $=389$, SRR $=2.17$ [CI, 2.02-2.34], $\mathrm{p}<0.001$ ).

Patients younger than 50 years and older than 80 years have higher rates of AKA after TKA (age $<50$ years $=367$ AKAs per 100,000 TKAs [1170 of 319,741], age 50-64 years $=129$ AKAs per 100,000 TKAs [2654 of 2,053,809], age 65-80 years $=141$ AKAs per 100,000 TKAs [4157 of $2,939,581]$, age $>80$ years $=337$ AKAs per 100,000 TKAs [1752 of 520,455], $\mathrm{p}<0.001)$. After adjusting for gender, race, and comorbidities, compared with patients of the age group 65 to 80 years, the adjusted rate of AKA was still higher in patients younger than age 50 years (adjusted rate $=473, \mathrm{SRR}=3.14[\mathrm{CI}, 2.94-3.36], \mathrm{p}<0.001)$ and older than 80 years (adjusted rate $=297$, SRR $=1.85[\mathrm{CI}$, 1.76-1.95], $\mathrm{p}<0.001)$. The differences in AKA rates by age were evident throughout the study period (Fig. 2). There was no significant decrease in the rates of AKA in any of the age groups (age $<50$ years, $\mathrm{p}=0.095$; age 50-64 years, $\mathrm{p}=0.252$; age 65-80 years, $\mathrm{p}=0.438$; age $>$ 80 years, $p=0.793$ ). The adjusted rate of AKA after septic complications was also higher in patients younger than age 50 years (adjusted rate $=353, \mathrm{SRR}=2.86$ [CI, 2.64-3.08], $\mathrm{p}<0.001$ ) and older than 80 years (adjusted rate $=235$, SRR $=1.76$ [CI, 1.67-1.86], $\mathrm{p}<0.001)$.

The annual number of AKAs increased by $107 \%$, from 522 procedures in 2000 to 1083 procedures in 2011. There was a significant increase in the number of AKAs from 2000 to 2011 (incidence rate ratio [IRR] = 1.06 [CI, 1.061.07], $\mathrm{p}<0.001)$. The rate of increase in AKA in the first half (2000-2005) was significantly higher than in the second half (2006-2011) (IRR first half versus second half: 1.10 [CI, 1.08-1.12] versus 1.01 [95\% CI, 1.00-1.02], $\mathrm{p}<$ 0.001). The mean annual standardized AKA rate was 169 AKAs per 100,000 TKAs (annual range, 133-201 AKAs per 100,000 TKAs) (Fig. 3). Linear regression analysis did not demonstrate a significant annual change in the standardized AKA rate $(\mathrm{p}=0.077)$. From 2000 to 2011, both septic and aseptic AKAs increased by $117 \%$ and $70 \%$, respectively. The annual septic burden during the study period ranged from $76 \%$ to $89 \%$ (average $=84 \%$ ). There was no significant year-to-year change in the septic burden over the study period $(\mathrm{p}=0.186)$.

\section{Discussion}

Even with expert, contemporary prosthetic approaches, AKA can result in severe impairment in quality of life [24],
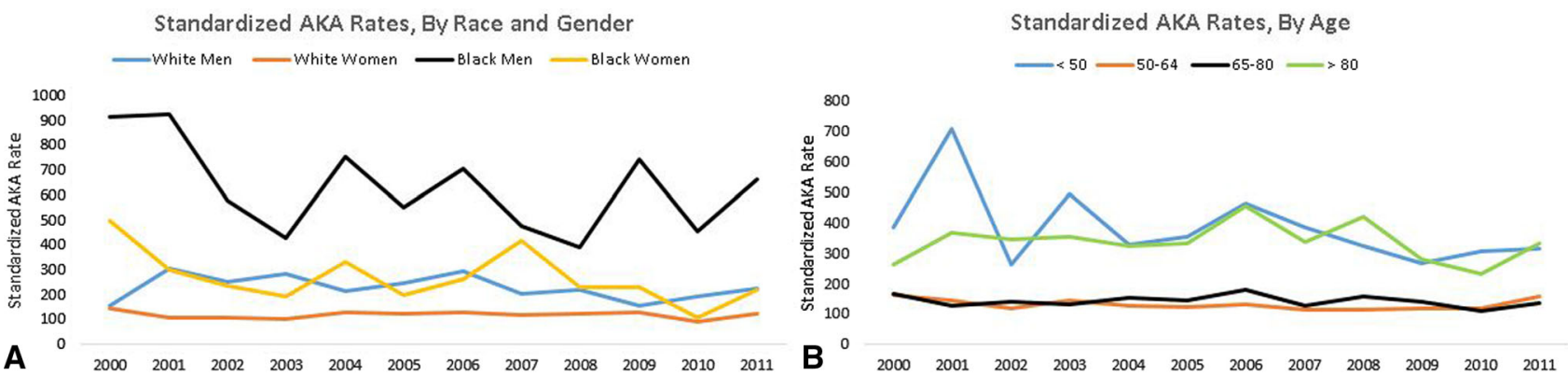

Fig. 2A-B The figure shows the annual changes in the standardized AKA rates (per 100,000 TKAs) for various gender-racial groups (A) and age groups (B) in the United States from 2000 to 2011. 


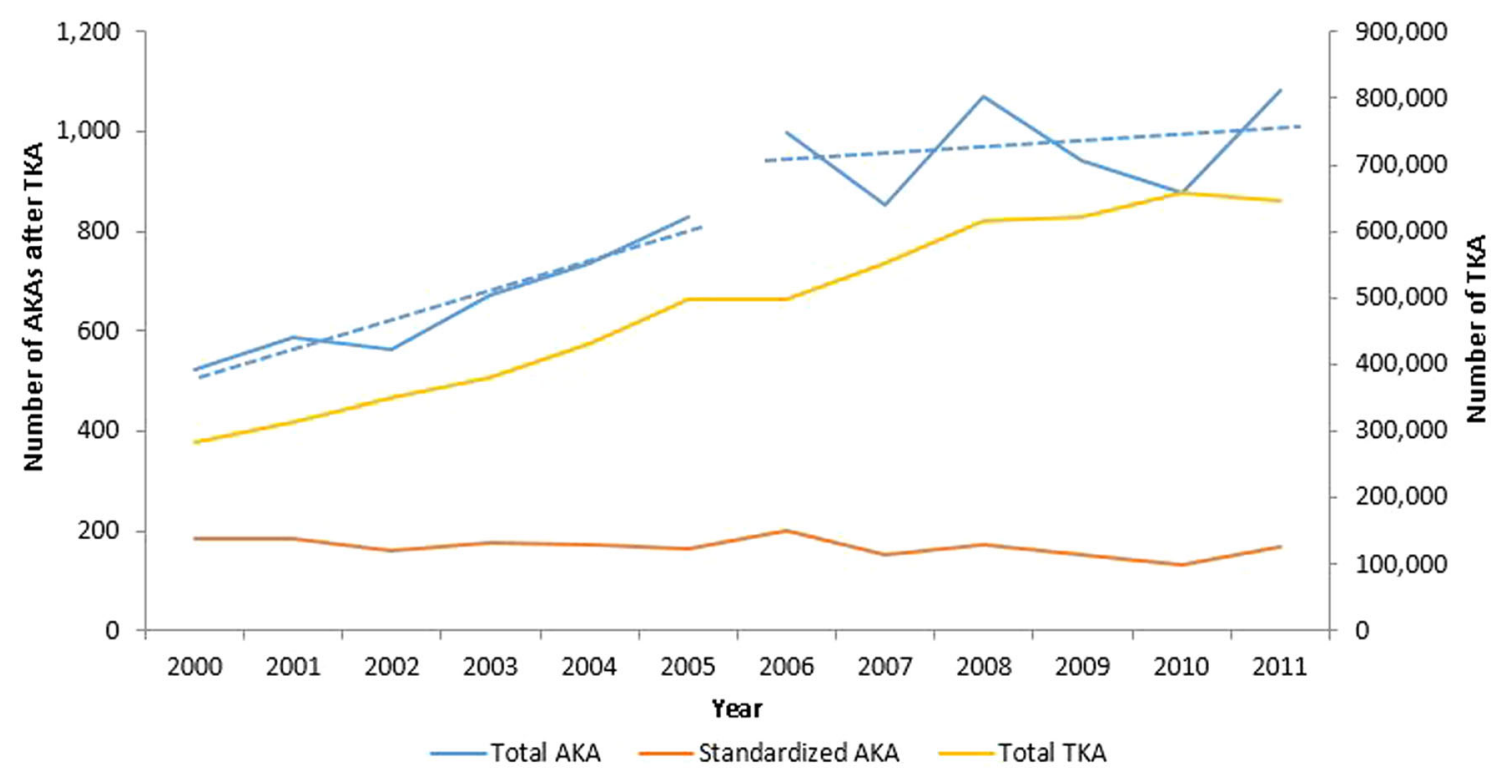

Fig. 3 The figure denotes the yearly changes in the number of AKAs, TKAs, and standardized AKA volume. The annual trends in AKA were split into two periods (2000-2005 and 2006-2011). The dotted

perhaps even more so among the older patients who experience it after TKA [7]. Because the number of AKAs after TKA is expected to rise further, it is important to explore the sociodemographic disparities in the rates of AKA after TKA. The present study evaluated whether racial and gender disparities existed in the rates of AKA using a nationally representative administrative database. The results of this study demonstrate variations in the distribution of AKA among various age, gender, and racial subgroups than expected based on the distribution of TKA.

The present study is primarily limited by the difficulty in accurately identifying AKA. National arthroplasty registries in countries like the United Kingdom, Canada, and Australia allow the monitoring of outcomes of various procedures at a national level [1,9]. However, as a result of the lack of such a national joint registry, an administrative database like NIS was used for estimating the national trends, in line with other investigators [3, 15]. Although NIS is the largest available national database, it is not without limitations. The NIS does not enable longitudinal tracking of individual patients and covers only short-term complications. Therefore, identifying a long-term complication like amputation, which develops 6 to 9 years after a TKA, was not directly feasible using NIS [6, 10, 26]. In addition, no ICD-9 procedure/diagnosis code was available for AKA. As a result, we relied on other available codes used for prosthetic joint/orthopaedic implant failure to estimate the incidence of amputations from failure of TKA. A detailed description of this approach is given (Appendix 1). Furthermore, any administrative database like the NIS is subject to the inaccuracies in coding, which is more lines (slopes) denote the rate of growth of AKA, which was significantly higher in the first half $(\mathrm{p}<0.001)$.

prevalent with comorbidities and diagnosis codes (as opposed to procedure codes) [19]. The NIS database was preferred over a state inpatient database or other databases because it was the largest available database, and the rarity of AKA necessitated the use of such a large database for reliable national trends. Additionally, the true risk of AKA after TKA cannot be identified from cross-sectional databases like NIS. The standardized rates reported in this study estimate the impact of underlying changes in TKA volume on the incidence of AKA and do not reflect the actual incidence rate of AKA after TKA.

Gender and racial disparities exist in the rates of AKA after TKA with the highest rates seen in black men. The reasons for these disparities are not fully understood and could be related to the differences in true risks of AKA, patient and surgeon preferences, and resource availability [5, 23]. Racial disparities in the level and rate of lower extremity amputations have been previously reported for etiologies like peripheral vascular disease and diabetes $[5,17]$. Holman et al. [8] reported that blacks are less likely to undergo attempts at limb salvage than their white counterparts treated for peripheral vascular disease. Further studies using longitudinal databases are required to understand the factors responsible for the racial differences observed in AKA after a failed TKA and to assess whether amputation is preferred to repeat revision TKA/arthrodesis in blacks.

Younger and older patients were found to have higher rates of AKA after TKA. The reason for this bimodal distribution in the rates of AKA could be related to the reasons for TKA in these age groups and the differences in 
comorbidities. Patients undergoing TKA at a younger age are likely to have diagnoses other than osteoarthritis and might have comorbidities that predisposed them to be candidates for TKA at a younger age like morbid obesity, rheumatoid arthritis, etc [12, 25]. Meehan et al. [20] showed that patients younger than 50 years are more likely to undergo revision as a result of PJI or aseptic mechanical failure at 1 year after TKA. Similarly, the higher rates of AKA in older populations could be the result of differences in comorbidities and/or patient preferences [13]. The higher rates in older patients could also be the result of the methodology used in this study. Because the study calculated rates by dividing the number of AKA in each group by the corresponding number of TKA, higher rates are expected for older patients because AKAs in the older age groups might have been for TKAs done at a younger age. Furthermore, although we adjusted for comorbidities using the Elixhauser score, there could be unadjusted confounders/comorbidities that might, at least in part, explain the age differences in the rates of AKA.

The present study did not find an increase in the rates of AKA during the study period. Therefore, the observed increase in AKA is probably the result of the rise in the demand for primary TKA without an accompanying decrease in the revision burden $[14,15]$. Also, the results of the present study suggest that the rate of increase in AKA diminished toward the later years of the study. This decline in the growth of AKA during the later years could be most likely the result of the stabilization in TKA growth in the later years. Although difficult to interpret based on the NIS data, it is possible that the flattening in the growth of AKA in later years could be the result of a decrease in the actual risk of AKA. In agreement with other studies, the majority of the amputations in this study were a result of septic complications of TKA $[10,26]$. With PJI being the major contributor to TKA-related amputations, efforts to reduce the amputations must be targeted at controlling infection.

Gender and racial disparities exist in the rates of AKA after TKA. These disparities were evident in the rates of AKA from both septic and aseptic complications of TKA. As a result of the personal, social, and economic impacts of AKAs, policymakers should be aware of the disparities in the rates of AKA after TKA and effective measures should be undertaken to address these disparities. Further research using longitudinal databases needs to be conducted to confirm the findings of this study and understand the potential reasons for these disparities.

\section{References}

1. Bohm ER, Dunbar MJ, Bourne R. The Canadian Joint Replacement Registry-what have we learned? Acta Orthop. 2010;81:119-121.
2. Centers for Disease Control and Prevention (CDC). Racial disparities in total knee replacement among Medicare enrolleesUnited States, 2000-2006. MMWR Morb Mortal Wkly Rep. 2009;58:133-138.

3. Cha MS, Cho SH, Kim DH, Yoon HK, Cho HS, Lee DY, Lee SH, Hwang SC. Two-stage total knee arthroplasty for prosthetic joint infection. Knee Surg Relat Res. 2015;27:82-89.

4. Dunlop DD, Manheim LM, Song J, Sohn M-W, Feinglass JM, Chang HJ, Chang RW. Age and racial/ethnic disparities in arthritis-related hip and knee surgeries. Med Care. 2008;46:200208.

5. Durazzo TS, Frencher S, Gusberg R. Influence of race on the management of lower extremity ischemia: revascularization vs amputation. JAMA Surg. 2013;148:617-623.

6. Fedorka CJ, Chen AF, McGarry WM, Parvizi J, Klatt BA. Functional ability after above-the-knee amputation for infected total knee arthroplasty. Clin Orthop Relat Res. 2011;469:10241032.

7. George J, Newman JM, Caravella JW, Klika AK, Barsoum WK, Higuera CA. Predicting functional outcomes after above knee amputation for infected total knee arthroplasty. J Arthroplasty. 2016 Aug 10. [Epub ahead of print]

8. Holman KH, Henke PK, Dimick JB, Birkmeyer JD. Racial disparities in the use of revascularization before leg amputation in Medicare patients. J Vasc Surg. 2011;54:420-426, 426.e1.

9. Huang T, Wang W, George D, Mao X, Graves S. What can we learn from AOANJRR 2014 annual report? Ann Transl Med. 2015;3:131.

10. Isiklar ZU, Landon GC, Tullos HS. Amputation after failed total knee arthroplasty. Clin Orthop Relat Res. 1994;299:173-178.

11. Jones CA, Pohar S. Health-related quality of life after total joint arthroplasty. Clin Geriatr Med. 2012;28:395-429.

12. Keeney JA, Nunley RM, Wright RW, Barrack RL, Clohisy JC. Are younger patients undergoing TKAs appropriately characterized as active? Clin Orthop Relat Res. 2014;472:12101216.

13. Kennedy JW, Johnston L, Cochrane L, Boscainos PJ. Total knee arthroplasty in the elderly: does age affect pain, function or complications? Clin Orthop Relat Res. 2013;471:19641969.

14. Kurtz S, Mowat F, Ong K, Chan N, Lau E, Halpern M. Prevalence of primary and revision total hip and knee arthroplasty in the United States from 1990 through 2002. J Bone Joint Surg Am. 2005;87:1487-1497.

15. Kurtz S, Ong K, Lau E, Mowat F, Halpern M. Projections of primary and revision hip and knee arthroplasty in the United States from 2005 to 2030. J Bone Joint Surg Am. 2007;89:780785 .

16. Lavernia CJ, Villa JM. Does race affect outcomes in total joint arthroplasty? Clin Orthop Relat Res. 2015;473:3535-3541.

17. Lefebvre KM, Lavery LA. Disparities in amputations in minorities. Clin Orthop Relat Res. 2011;469:1941-1950.

18. Lentino JR. Prosthetic joint infections: bane of orthopedists, challenge for infectious disease specialists. Clin Infect Dis. 2003;36:1157-1161.

19. Losina E, Barrett J, Baron JA, Katz JN. Accuracy of Medicare claims data for rheumatologic diagnoses in total hip replacement recipients. J Clin Epidemiol. 2003;56:515-519.

20. Meehan JP, Danielsen B, Kim SH, Jamali AA, White RH. Younger age is associated with a higher risk of early periprosthetic joint infection and aseptic mechanical failure after total knee arthroplasty. J Bone Joint Surg Am. 2014;96:529-535.

21. Mozella A de P, da Palma IM da, Souza AF de, Gouget GO, Cobra HA de AB. Amputation after failure or complication of total knee arthroplasty: prevalence, etiology and functional outcomes. Rev Bras Ortop. 2013;48:406-411. 
22. Nationwide Inpatient Sample (NIS). Healthcare Cost and Utilization Project (HCUP). 2000-2011. Rockville, MD, USA: Agency for Healthcare Research and Quality; 2013.

23. Peek ME. Gender differences in diabetes-related lower extremity amputations. Clin Orthop Relat Res. 2011;469:1951-1955.

24. Pell JP, Donnan PT, Fowkes FG, Ruckley CV. Quality of life following lower limb amputation for peripheral arterial disease. Eur J Vasc Surg. 1993;7:448-451.

25. Shah S, Schwartz B, Schwartz A, Goldberg B, Chmell S. Total knee arthroplasty in the younger patient. J Knee Surg. 2016 Oct 24. [Epub ahead of print]
26. Sierra RJ, Trousdale RT, Pagnano MW. Above-the-knee amputation after a total knee replacement: prevalence, etiology, and functional outcome. J Bone Joint Surg Am. 2003;85:1000-1004.

27. Skou ST, Roos EM, Laursen MB, Rathleff MS, Arendt-Nielsen L, Simonsen O, Rasmussen S. A randomized, controlled trial of total knee replacement. $N$ Engl J Med. 2015;373:1597-1606.

28. Zhang W, Lyman S, Boutin-Foster C, Parks ML, Pan T-J, Lan A, Ma Y. Racial and ethnic disparities in utilization rate, hospital volume, and perioperative outcomes after total knee arthroplasty. J Bone Joint Surg Am. 2016;98:1243-1252. 